\title{
Electric Field Simulation and Effect of Different Solvent Ratios on the Performance of Single Electrospun PVDF/PEI Composite Film
}

\author{
Jin-gang Jiang, ${ }^{1}$ Zhao Wang, ${ }^{1}$ Hong-wei Duan, ${ }^{2}$ Jing-qiang Liu, ${ }^{3}$ and Xiao-wei Guo' \\ ${ }^{1}$ Intelligent Machine Institute, Harbin University of Science and Technology, Xuefu Road No. 52, Nangang District, \\ Harbin 150080, China \\ ${ }^{2}$ The Graduate School, Harbin University of Science and Technology, Xuefu Road No. 52, Nangang District, Harbin 150080, China \\ ${ }^{3}$ School of Material Science and Engineering, Harbin University of Science and Technology, Xuefu Road No. 52, \\ Nangang District, Harbin 150080, China
}

Correspondence should be addressed to Jin-gang Jiang; jiangjingang@hrbust.edu.cn

Received 12 April 2016; Accepted 29 August 2016

Academic Editor: Wei Zhou

Copyright (C) 2016 Jin-gang Jiang et al. This is an open access article distributed under the Creative Commons Attribution License, which permits unrestricted use, distribution, and reproduction in any medium, provided the original work is properly cited.

On the basis of the finite element calculation theory of electric field, the electric field distribution in a representative electrospinning device is computed. The electric field structure of a needle-plate type electrospinning device was simulated by means of ANSYS software. And the vector distribution of the nozzle on the spinneret pipe was got. For the purpose of the analysis on the influence of different solvent ratios on the performance of a single electrospun PVDF/PEI composite film, polyvinylidene fluoride and polyetherimide with a mass ratio of $8 / 2$ were dissolved in a mixed solvent. The mixed solvent is composed of $\mathrm{N}, \mathrm{N}$-dimethylformamide and tetrahydrofuran, added in different proportions. Through the electrostatic spinning technology, PVDF/PEI composite fiber membranes were prepared. Using scanning electron microscopy (SEM), X-ray diffraction (XRD), differential scanning calorimetry (DSC), and mechanical properties testing, the effects of tetrahydrofuran on the composite microstructure, crystallinity, and mechanical properties of the PVDF/PEI composite fiber membranes are discussed.

\section{Introduction}

Electrospinning is a procedure of generating continuous nanofibers using polymer solutions in the presence of an electric field [1-3]. Nanofibrous nonwovens, because of interconnected pores and a large surface-to-volume ratio, enable many applications of these structures in the field of filtration media, medicine, and industry $[4,5]$. It is crucial to simulate the electric field distribution of an electrospinning device [6-9]. For electrospinning, a metallic capillary with a high voltage DC is joined to a container, which is filled with a polymer solution $[10,11]$. When the electric field force is higher than the surface tension of the polymer solution, a thin jet is formed in the capillary nozzle. Figure 1 is a schematic illustration of an electrospinning experimental system. The distribution of electric field directly influences the fineness and morphology of the electrospun fiber [1216]. The polyvinyl chloride insulating tubes are used to form a unified and centralized electric field [7]. COMSOL multiphysics software was employed to simulate and analyze the value and distribution of the electric field strength during a multineedle electrospinning process [2]. The metal needle length under the metal plate electrode is changed to create different electric field distributions [8]. Electric field distribution of the multijet spinning device is improved [17]. A new electrospinning configuration with a 19-hole multistep has been demonstrated [18]. Other related studies on electrospinning are given in [19-22]. Electric field distribution affects fiber alignment and further affects the performance of electrospun composite film. Consequently, it is crucial to simulate the electric field distribution in an electrospinning device and to analyze the effect of different solvent 
ratios on the performance of a single electrospun PVDF/ PEI composite film.

\section{Computational Principles of Electric Field}

2.1. Potential Function $\varphi$. Assuming that the potential function $\varphi$ of each triangular mesh element $e$ is linear for the variables $r$ and $z$ in the cylindrical coordinate, then the electric field is perceived as approximate homogeneous in each triangular mesh element domain. So, the potential of every point of each triangular mesh element follows such a rule

$$
\varphi=\alpha_{1}+\alpha_{2} r+\alpha_{3} z
$$

where $\alpha_{1}, \alpha_{2}$, and $\alpha_{3}$ are the coefficients and $r$ and $z$ are the distance between the center of triangular mesh element to $z$ axis and the coordinate value of the center of the triangular mesh element along with $z$-axis, respectively.

$i, j$, and $m$ represent node numbers of single triangular mesh element, respectively. Therefore,

$$
\begin{aligned}
\varphi_{i} & =\alpha_{1}+\alpha_{2} r_{i}+\alpha_{3} z_{i} \\
\varphi_{j} & =\alpha_{1}+\alpha_{2} r_{j}+\alpha_{3} z_{j} \\
\varphi_{m} & =\alpha_{1}+\alpha_{2} r_{m}+\alpha_{3} z_{m} \\
\alpha_{1} & =\frac{1}{2 S_{e}}\left(\alpha_{i} \varphi_{i}+\alpha_{j} \varphi_{j}+\alpha_{m} \varphi_{m}\right) \\
\alpha_{2} & =\frac{1}{2 S_{e}}\left(b_{i} \varphi_{i}+b_{j} \varphi_{j}+b_{m} \varphi_{m}\right) \\
\alpha_{3} & =\frac{1}{2 S_{e}}\left(c_{i} \varphi_{i}+c_{j} \varphi_{j}+c_{m} \varphi_{m}\right),
\end{aligned}
$$

where

$$
\begin{aligned}
\alpha_{i} & =r_{i} z_{m}-r_{m} z_{j} \\
\alpha_{j} & =r_{m} z_{i}-r_{i} z_{m} \\
\alpha_{m} & =r_{i} z_{j}-r_{j} z_{i} \\
b_{i} & =z_{j}-z_{m} \\
b_{j} & =z_{m}-z_{i} \\
b_{m} & =z_{i}-z_{j} \\
c_{i} & =r_{m}-r_{j} \\
c_{j} & =r_{i}-r_{m} \\
c_{m} & =r_{j}-r_{i} .
\end{aligned}
$$

The area $S_{e}$ of triangular mesh element $e$ is as follows:

$$
S_{e}=\frac{1}{2}\left|\begin{array}{ccc}
1 & r_{i} & z_{i} \\
1 & r_{j} & z_{j} \\
1 & r_{m} & z_{m}
\end{array}\right|=\frac{1}{2}\left(b_{i} c_{j}-b_{j} c_{i}\right) .
$$

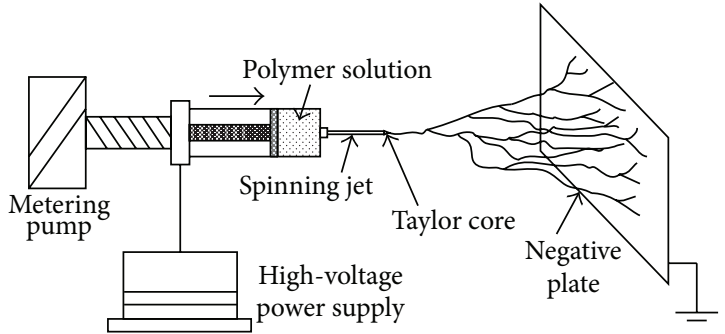

FIGURE 1: Electrospinning device.

Hence, the interpolating functions of triangular mesh element $e$ can be expressed as

$$
\begin{aligned}
& \varphi(x, y)=\frac{1}{2 S_{e}}\left[\left(\alpha_{i}+b_{i} r+c_{i} z\right) \varphi_{i}+\left(\alpha_{j}+b_{j} r+c_{j} z\right) \varphi_{j}\right. \\
& \left.+\left(\alpha_{m}+b_{m} r+c_{m} z\right) \varphi_{m}\right] .
\end{aligned}
$$

2.2. Energy Function of Triangular Mesh Element e. Energy function of triangular mesh element $e$ is as follows:

$$
W_{e}=\iint_{e} \frac{\varepsilon_{e}}{2}\left[\left(\frac{\partial \varphi^{3}}{\partial r}+\frac{\partial \varphi^{2}}{\partial z}\right) \cdot 2 \pi d_{r} d_{z}\right]
$$

where $\varepsilon_{e}$ is the dielectric constant of triangular mesh element e.

According to (3), we know that $\partial \varphi / \partial r=\alpha_{2}, \partial \varphi / \partial z=\alpha_{3}$; that is, $\partial \varphi / \partial r$ and $\partial \varphi / \partial z$ of each point in triangular mesh element $e$ are both constant. And they are not affected by coordinate value $(r, z)$. Therefore, simplified $W_{e}$ is as follows:

$$
\begin{aligned}
& W_{e} \\
& =\frac{\varepsilon_{e}}{2} \\
& \quad \cdot 2 \pi \frac{\left(\sum_{s=i, j, m} b_{s} \varphi_{s}\right)^{2}+\left(\sum_{s=i, j, m} c_{s} \varphi_{s}\right)^{2}}{4 S_{e}^{2}} \iint_{s} r d_{r} d_{z} \\
& \iint_{s} r d_{r} d_{z}=\frac{r_{i}+r_{j}+r_{m}}{3} S_{e}=r_{e} S_{e},
\end{aligned}
$$

where $r_{e}$ represents the distance between the center of triangular mesh element $e$ and $z$-axis.

Therefore,

$$
W_{e}=\frac{1}{2} \cdot \frac{2 \pi \varepsilon_{e} r_{e}}{4 S_{e}}\left[\left(\sum b_{s} \varphi_{s}\right)^{2}+\left(\sum c_{s} \varphi_{s}\right)^{2}\right] .
$$

2.3. Calculation of the Electric Field. Electric field strength is as follows:

$$
\vec{E}=-\nabla \varphi=-\frac{d \varphi}{d r} \cdot \overrightarrow{e_{r}}-\frac{d \varphi}{d z} \cdot \overrightarrow{e_{r}}=E_{r e} \overrightarrow{e_{r}}+E_{z e} \overrightarrow{e_{r}}
$$




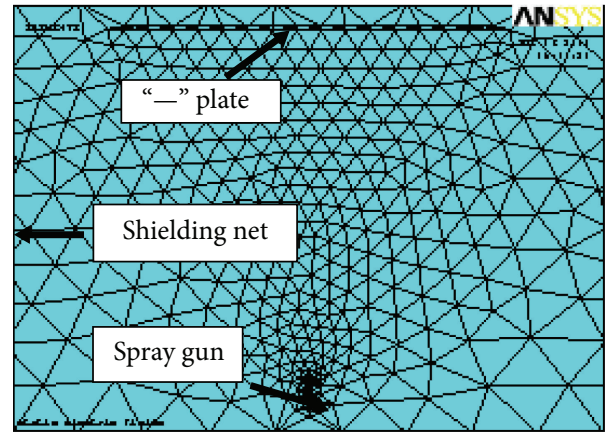

FIgURE 2: Simplified FEA mesh model.

TABLE 1: Parameters setting.

\begin{tabular}{lcc}
\hline & Dielectric constant & Voltage load \\
\hline Spray gun & 2 & $8000 \mathrm{~V}$ \\
“-” plate & 2 & $-5000 \mathrm{~V}$ \\
Air medium & 1 & - \\
Shielding net & 5 & $0 \mathrm{~V}$ \\
\hline
\end{tabular}

$E_{r e}$ and $E_{z e}$ are $r$-component and $z$-component of the electric field strength, respectively:

$$
\begin{aligned}
& E_{r e}=-\frac{\partial \varphi}{\partial r}=-\frac{1}{2 S_{e}}\left(\sum_{s=i, j, m} b_{s} \varphi_{s}\right) \\
& E_{z e}=-\frac{\partial \varphi}{\partial z}=-\frac{1}{2 S_{e}}\left(\sum_{s=i, j, m} c_{s} \varphi_{s}\right) .
\end{aligned}
$$

Its absolute value is

$$
E=\sqrt{E_{r e}^{2}+E_{z e}^{2}}=\sqrt{\frac{\partial \varphi}{\partial r}+\frac{\partial \varphi}{\partial z}} .
$$

\section{Electric Field Simulation of Needle-Plate Type Electrospinning Machine}

3.1. Finite Element Analysis (FEA) of Electric Field. On the basis of the $H$-method for the FEA of the electric field [2, 3], using ANSYS software, we proposed some assumptions: (1) the dielectric constant, which is not affected by the electric field, is definite value; (2) electrification of the spinning fiber has no influence on the electric charge; (3) the control system has no influence on the electric field distribution; (4) the volume density of the field electric charge is $\rho=0$. By comparing with different kinds of mesh element, eight nodal points PLANE121 are selected. Figure 2 is a simplified FEA mesh model using ANSYS software. Table 1 shows the parameters setting.

3.2. Simulation Result of the Electric Field. The vector distribution of the electric field, which has an axial symmetric characteristic, is shown in Figure 3. Figure 4 shows that

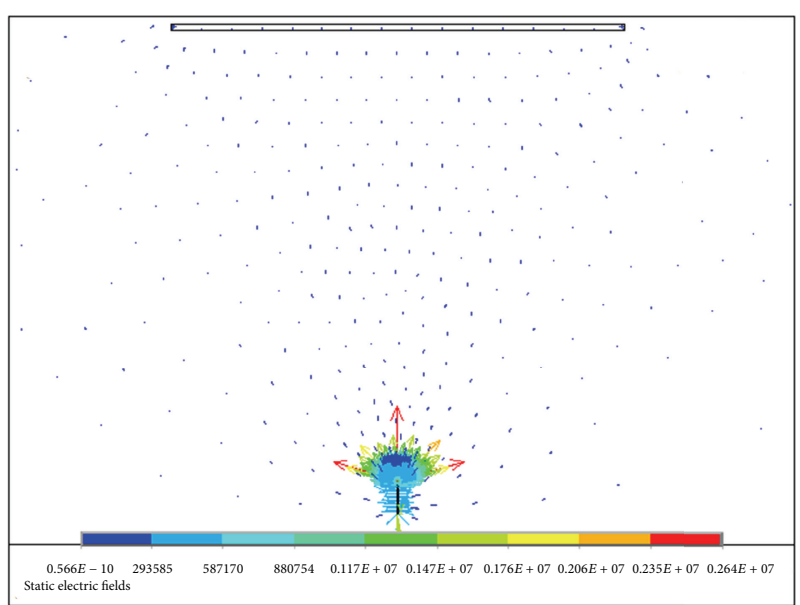

FIGURE 3: Vector distribution of the electric field intensity.

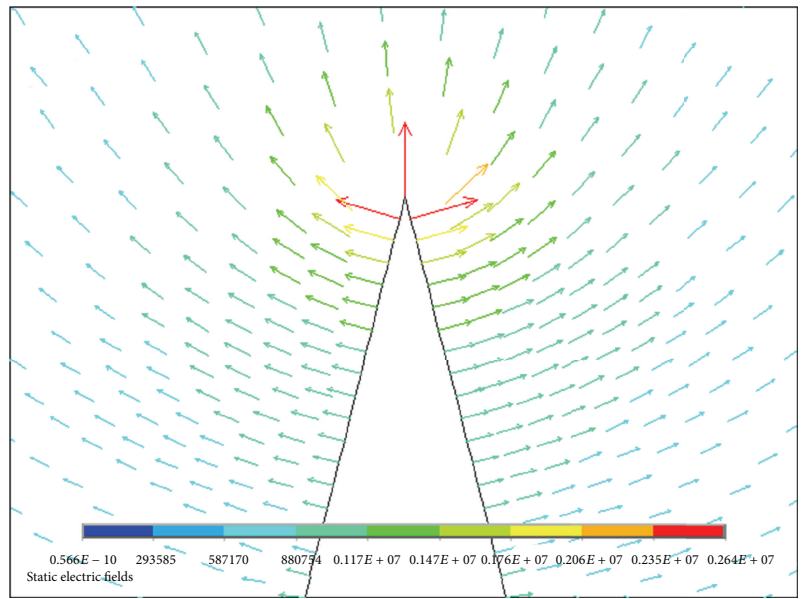

FIGURE 4: Vector distribution of nozzle.

the peak of the electric field intensity appears at the spinning pipe orifice, and the direction of the electric field points to the negative plate. The peak of the electric field intensity is $E=2.64 \times 10^{6} \mathrm{~V} / \mathrm{m}$. Influence of the collecting distance on the electric field intensity is shown in Figure 5. We determined that the field intensity suddenly went down to the minimum value, particularly at a near distance with spinneret pipe orifice.

\section{Electrospinning Experiment}

4.1. Reagent. PVDF (Arkema SA, Mw $=3 \times 10^{5}$ ) and PEI (GE Ultem-1000) were used without further purification to prepare the electrospun fibrous materials. N,N-Dimethylformamide (DMF, >99.5\%), absolute ethanol, and tetrahydrofuran (THF) were purchased from Tianjin Fu Yu Hua Gong Co., Ltd.

4.2. Electrospinning Experimental System. Figure 6 is the electrospinning experimental system. The high voltage DC power supply, fabricated at the Electrical Engineering Department 
$\left(\times 10^{3}\right)$

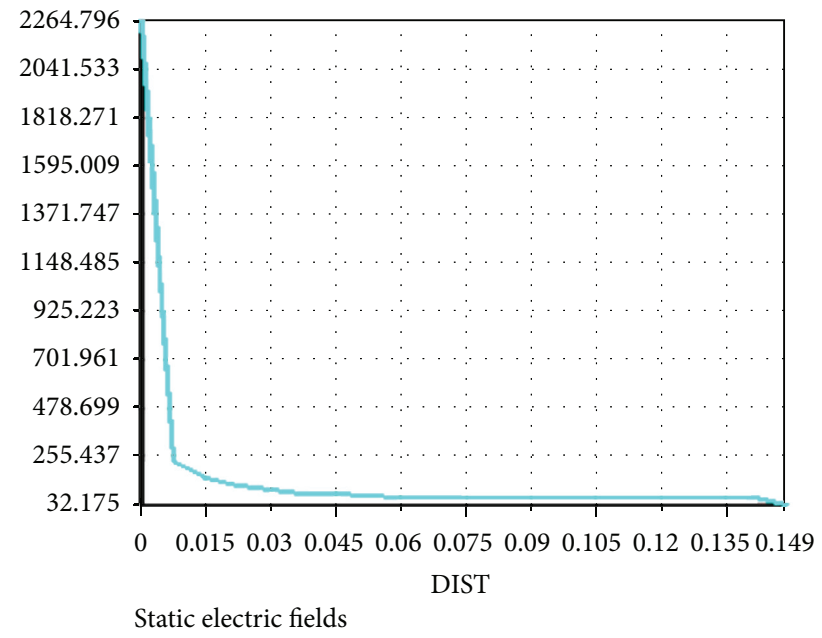

Figure 5: Influence of the collecting distance on the electric field intensity.

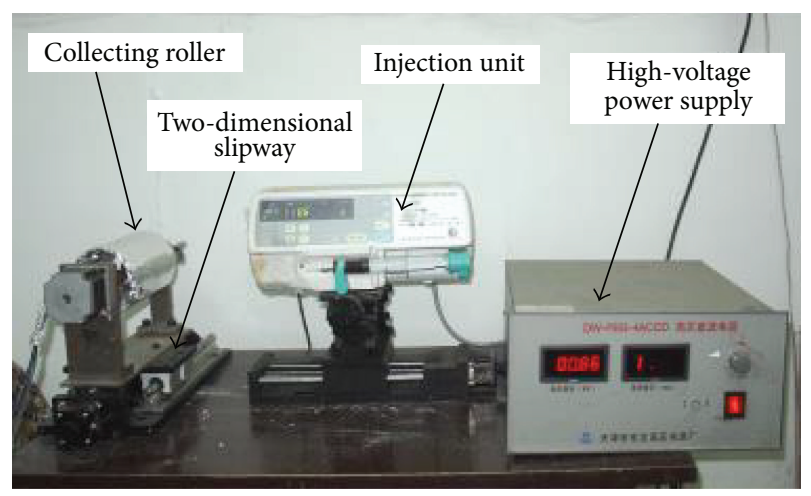

FIGURE 6: Electrospinning experimental system.

of Tsinghua University, can be continuously adjusted between $0 \mathrm{~V}$ and $100 \mathrm{KV}$. In order to avoid the influence on the input signal, the electrospinning experimental system chooses shielded cable as the connecting line.

4.3. Preparation of PVDF/PEI Composite Fiber Membrane. To prepare the electrospinning solution with a mass fraction of $18 \%$, the polymer with a mass ratio of $\mathrm{m}(\mathrm{PVDF}) / \mathrm{m}(\mathrm{PEI})=8 / 2$ was dissolved in the mixed solvent, with $\mathrm{m}(\mathrm{DMF}) / \mathrm{m}$ (THF) of $9 / 1,8 / 2,7 / 3,6 / 4$, and $5 / 5$. At room temperature $20^{\circ} \mathrm{C}$, the electrospinning process parameters are set to the static voltage $16 \mathrm{KV}$ and the flow rate is $0.1 \mathrm{~mL} / \mathrm{h}$. A static collecting device was used. The distance, which is between the nozzle and the collecting device, is $20 \mathrm{~cm}$. The thickness of the PVDF/PEI composite fiber membrane was controlled to 40$80 \mu \mathrm{m}$. The finished PVDF/PEI composite fiber membrane is thermally treated at $80^{\circ} \mathrm{C}$ for $12 \mathrm{~h}$, and then it is packed until used.

4.4. Scanning Electron Microscopy (SEM). After spraying the sample, the surface microscopic morphology of the
PVDF/PEI composite fiber membrane was observed using a FEI Sirion200 SEM (Philips Corporation, Netherlands).

4.5. X-Ray Diffraction (XRD). The composite fiber membranes were tested by XRD (PANalytical Corporation, Netherlands). The diffraction patterns of the composite fiber membranes were analyzed using a $\mathrm{Cu}(\mathrm{K})$ target. The tube voltage was $40 \mathrm{KV}$, and the tube current was $50 \mathrm{~mA}$. The scanning speed is $1^{\circ} / \mathrm{min}$ over the scanning angle range of $5^{\circ}$ to $80^{\circ}$, with a step length of $0.02^{\circ}$.

4.6. Differential Scanning Calorimetry (DSC). Thermal properties of the composite fiber membrane are measured under $\mathrm{N}_{2}$ using DSC. The initial temperature was $40^{\circ} \mathrm{C}$, and the composite fiber membranes were heated to $250^{\circ} \mathrm{C}$ at the rate of $10^{\circ} \mathrm{C} / \mathrm{min}$. Through the change in the height of the endothermic peak, the area under the endothermic peak and the enthalpy value and the influence of the crystallinity of the fiber membrane on the mechanical properties are analyzed. The crystallinity can be calculated as follows:

$$
X_{c}=\frac{\Delta H_{f}}{\Delta H_{c}} \times 100 \%,
$$

where $\Delta H_{f}$ is the melting enthalpy of the samples and $\Delta H_{c}$ is the melting enthalpy of the fully crystallized PVDF $(104.7 \mathrm{~J} / \mathrm{g})$.

4.7. Mechanical Properties. The tensional strength and elongation ratio of the composite fiber membrane are tested using the AGS-J electronic universal testing machine (Shimadzu Corporation, Japan), according to the standard of the 11th chapter of 13542.2-2009 GB/T. The finished composite fiber membrane was cut to a length of $10 \mathrm{~cm}$ and a width of $1 \mathrm{~cm}$, and the distance between the fixtures was $100 \mathrm{~mm}$. The tensile test is performed at a tensile rate of $5 \mathrm{~mm} / \mathrm{min}$ along the direction of the sample length. The tensile strength can be calculated as follows:

$$
\delta=\frac{G}{S}
$$

where $\delta$ is the tensile strength, $G$ is the sample load, and $S$ is the cross-sectional area. Each composite fiber membrane has three samples, and the average value is taken.

\section{Discussion}

5.1. Surface Topography. From the SEM photo of the PVDF/ PEI composite fiber membrane at 5000x magnification, which is shown in Figure 7, it can be clearly seen that with the increase in the proportion of THF the morphology of the fibers is obviously different. When the solvent mass ratio $\mathrm{m}(\mathrm{DMF}) / \mathrm{m}(\mathrm{THF})$ is $9 / 1$, the fibers intertwist, and the fibers have more cross-linking branches, as shown in Figure 7(a). The morphological uniformity decreases because the volatility of THF and DMF is different, and when the THF content is less, the electrospinning solution has poor volatility. Under the influence of the electric field force, THF volatilizes rapidly. 


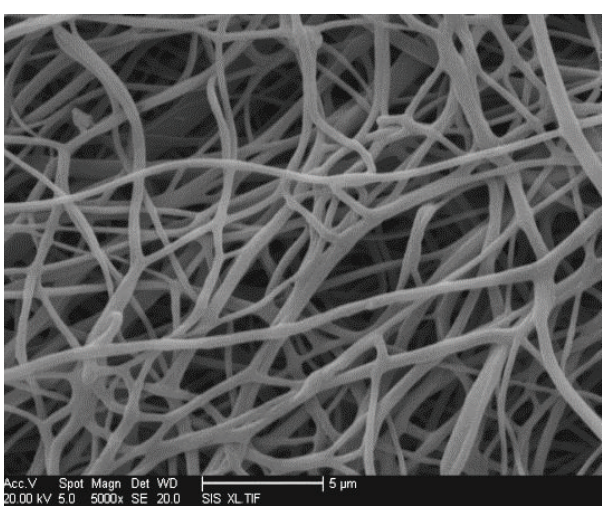

(a)

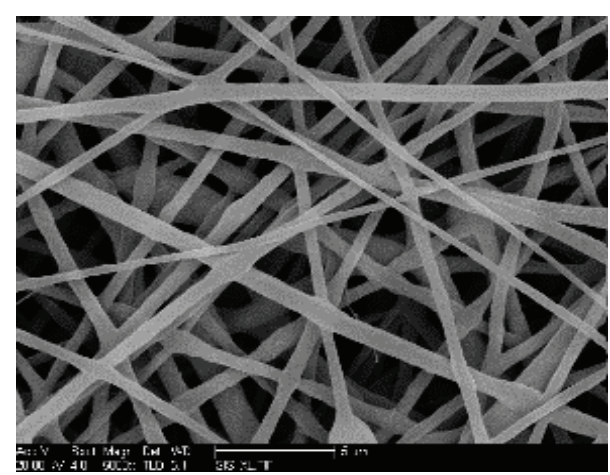

(c)

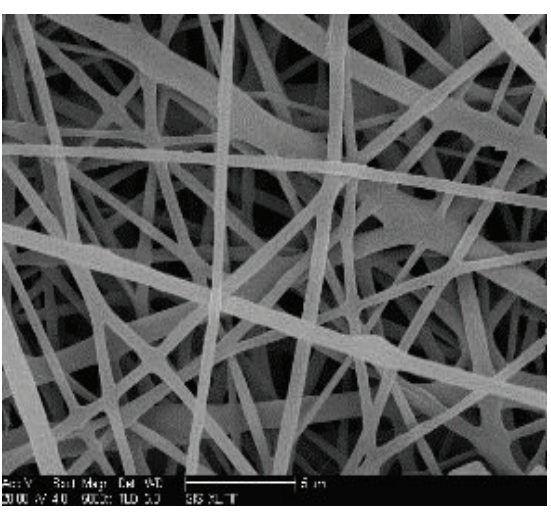

(b)

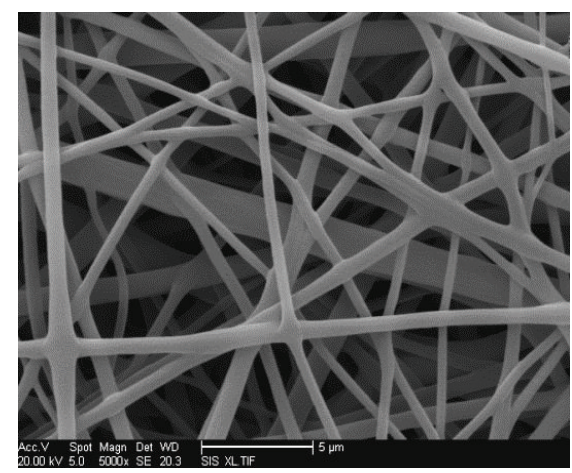

(d)

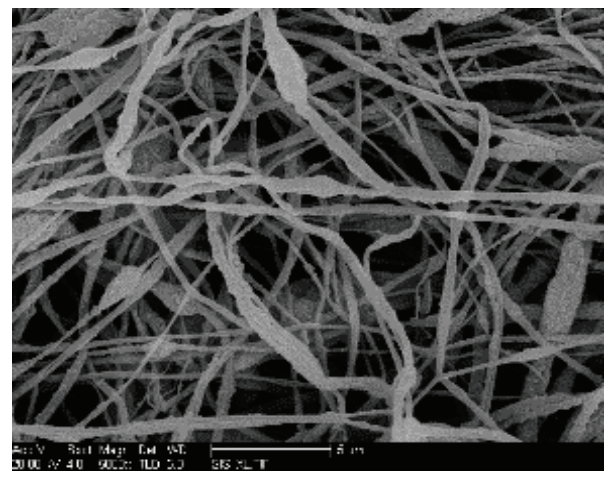

(e)

FIGURE 7: SEM photos of PVDF/PEI composite fiber membrane prepared by electrospinning with the different solvent mass ratio of $\mathrm{m}(\mathrm{DMF}) / \mathrm{m}$ (THF). (a) m(DMF)/m(THF): 9/1; (b) m(DMF)/m(THF): 8/2; (c) m(DMF)/m(THF): 7/3; (d) m(DMF)/m(THF): 6/4; (e) $\mathrm{m}(\mathrm{DMF}) / \mathrm{m}$ (THF): 5/5.

This causes the jet to solidify into fibers at a slower rate, and the jet has not yet solidified after deposition in the collection device, and the nonsolidified fibers are dissolved in the residual solvent, which causes the phenomenon of intertwist between the fibers. When the solvent mass ratios of $\mathrm{m}(\mathrm{DMF}) / \mathrm{m}(\mathrm{THF})$ are $8 / 2,7 / 3$, and $6 / 4$, with an increase in the THF content in the solvent system, the solvent volatility increases, the surface tension of the solution decreases, and the volatile component (THF) in the mixed solvent increases. This is beneficial to the formation and consolidation of PVDF/PEI fibers. As can be seen from Figures 7(b), 7(c), and $7(\mathrm{~d})$, the PVDF/PEI composite fiber membranes are typical interpenetrating microporous structures with no droplets or bead defects. When the solvent mass ratio $\mathrm{m}(\mathrm{DMF}) / \mathrm{m}(\mathrm{THF})$ is $7 / 3$, the fiber diameter distribution is relatively uniform. In Figure $7(\mathrm{e})$, when the solvent mass ratio $\mathrm{m}(\mathrm{DMF}) / \mathrm{m}(\mathrm{THF})$ is $5 / 5$, the beads appear on the fibers, and the fiber diameter distribution is relatively wide. This is because when the volatile solvent (THF) content is too large, the solution can volatilize easily, so it is difficult to form a Taylor cone at the nozzle. It is not easy to form a stable electrospinning jet, and the molecular chain is short and not sufficient for entanglement. So this will cause the formation of beads on the fiber. 


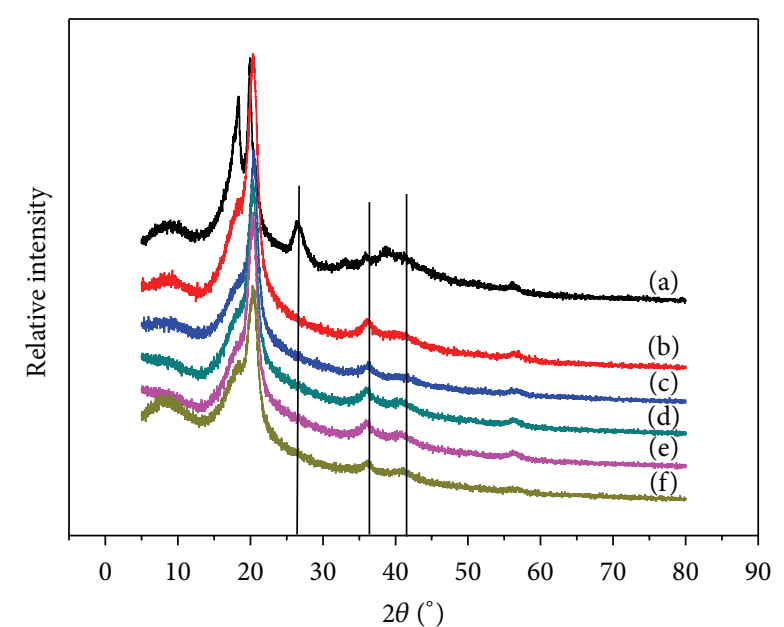
(a) Pure PVDF power
(d) $\mathrm{m}(\mathrm{DMF}) / \mathrm{m}(\mathrm{THF})=7 / 3$
(b) $\mathrm{m}(\mathrm{DMF}) / \mathrm{m}(\mathrm{THF})=9 / 1$
(e) $\mathrm{m}(\mathrm{DMF}) / \mathrm{m}(\mathrm{THF})=6 / 4$
(c) $\mathrm{m}(\mathrm{DMF}) / \mathrm{m}(\mathrm{THF})=8 / 2$
(f) $\mathrm{m}(\mathrm{DMF}) / \mathrm{m}(\mathrm{THF})=5 / 5$

FIgure 8: The X-ray diffractogram of PVDF/PEI composite fiber membrane prepared by electrospinning with different solvent ratios.

5.2. XRD Analysis. Figure 8 shows the $\mathrm{X}$-ray diffractogram of PVDF/PEI composite fiber membranes prepared by electrospinning with different solvent ratios. In the diffractogram of the PVDF powder sample without electric field polarization, there are obvious characteristic peaks at a diffraction angle of $27^{\circ}$, which corresponds to the $\alpha$ phase and belongs to the monoclinic system. However, the corresponding curve for the PVDF/PEI composite fiber membrane with different solvent ratios prepared by electrospinning becomes flat and even disappears compared to the peak at $27^{\circ}$. This shows that the $\alpha$ crystal form of the PVDF/PEI composite fiber membrane gradually decreases compared to the pure PVDF fiber membrane. In addition, compared to the pure PVDF powder, the PVDF/PEI composite fiber membrane shows peaks at $37^{\circ}$ and $42^{\circ}$, which correspond to the $\delta$ and $\beta$ phases, respectively. However, these two characteristic peaks are very weak in pure PVDF powder. Although the intensity of the two peaks in the PVDF/PEI composite fiber membrane is not very high, it can be attributed to the change in the crystal form of PVDF during electrospinning. The $\alpha$ crystal form is transformed into the $\beta$ and $\delta$ crystal forms, increasing their content in the PEI/PVDF composite fiber membrane. Under the influence of the polarized electric field, the PVDF will produce a phase transition from $\alpha$ to $\beta$. The reason for the transformation from nonpolar to the polar $\beta$ crystal phase is the orientation of the dipole charge along the electric field direction, the rotation of the PVDF molecular chain, and the orientation of the intrinsic dipole, which leads to the full trans-planar zigzag conformation, changing the PVDF crystal type.

5.3. DSC Analysis. The DSC curves of different solvent ratio PVDF/PEI composite fiber membranes prepared by

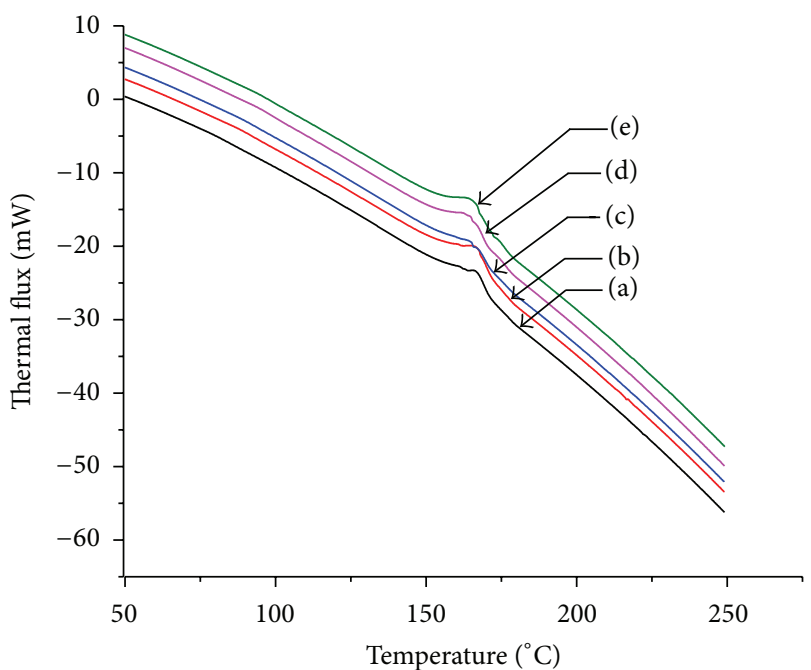
(a) $\mathrm{m}(\mathrm{DMF}) / \mathrm{m}(\mathrm{THF})=9 / 1$
(b) $\mathrm{m}(\mathrm{DMF}) / \mathrm{m}(\mathrm{THF})=8 / 2$
(d) $\mathrm{m}(\mathrm{DMF}) / \mathrm{m}(\mathrm{THF})=6 / 4$
(c) $\mathrm{m}(\mathrm{DMF}) / \mathrm{m}(\mathrm{THF})=7 / 3$
(e) $\mathrm{m}(\mathrm{DMF}) / \mathrm{m}(\mathrm{THF})=5 / 5$

FIGURE 9: The DSC curves of different solvent ratio PVDF/PEI composite fiber membranes prepared by electrospinning.

TABLE 2: The thermal properties and the crystallinity of different solvents ratio PVDF/PEI composite fiber membrane prepared by electrospinning.

\begin{tabular}{|c|c|c|c|c|c|}
\hline \multirow{2}{*}{ Sample } & \multicolumn{5}{|c|}{$\mathrm{m}(\mathrm{DMF}) / \mathrm{m}(\mathrm{THF})$} \\
\hline & $9 / 1$ & $8 / 2$ & $7 / 3$ & $6 / 4$ & $5 / 5$ \\
\hline Melting point $/{ }^{\circ} \mathrm{C}$ & 166.59 & 165.21 & 165.07 & 164.66 & 166.58 \\
\hline $\begin{array}{l}\text { Melting } \\
\text { enthalpy } /\left(\Delta H_{f}\right) / \mathrm{J} \cdot \mathrm{g}^{-1}\end{array}$ & 25.77 & 28.16 & 32.74 & 29.20 & 27.08 \\
\hline Crystallinity $/ \%$ & 24.61 & 26.89 & 31.27 & 27.88 & 25.86 \\
\hline
\end{tabular}

electrospinning are shown in Figure 9, and the thermal properties and the crystallinity are shown in Table 2.

The melting point of PVDF is about $170^{\circ} \mathrm{C}$, so this material, which is reflected by the endothermic peak, is the PVDF fiber. The initial, end, and the peak position of the endothermic peak are located within the allowable error scope. Table 2 shows the thermal properties and the crystallinity of different solvents ratio PVDF/PEI composite fiber membrane prepared by electrospinning. The melting point of the PVDF/PEI composite fiber membrane with different solvent ratios prepared by electrospinning did not change. However, with an increase in the proportion of THF, the crystallinity of the PVDF/PEI composite fiber films increased first and, then, decreased. When the mass ratio of DMF/THF was $7 / 3$, the crystallinity was 31.27 . This shows that under the electric field force crystallization changes are more likely to occur in PVDF because of the interaction of the dipole and the effect of the hydrogen bond of the chain between the DMF molecule, THF molecule, and PVDF. However, with the increase in the content of the volatile solvent, THF, the spinning solution became stronger. When 


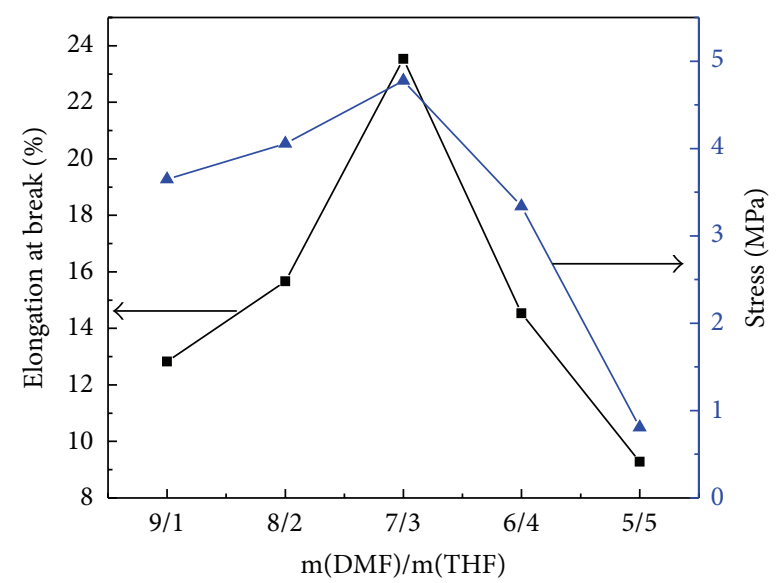

FIGURE 10: The mechanical properties of different solvent ratio PVDF/PEI composite fiber membranes prepared by electrospinning.

the THF content is too large, it leads to instability of the jet and a decrease in the crystallinity of the PVDF/PEI composite fiber films.

5.4. Mechanical Properties Analysis. The mechanical properties of different solvent ratio PVDF/PEI composite fiber membranes prepared by electrospinning are shown in Figure 10. With the increase in the THF content, the fracture stress and elongation at break first increase and, then, decrease. When the $\mathrm{m}(\mathrm{DMF}) / \mathrm{m}(\mathrm{THF})$ is equal to $7: 3$, the fracture stress and elongation at break of the composite fiber membranes reach the maximum values of $4.778 \mathrm{MPa}$ and $23.5327 \%$, respectively. This is because the solution forms a jet during the process of electrospinning, and the polymer molecular chain opened in the solvent is highly stretched by the electric field force. The polymer molecular chain is oriented and arranged again. With the evaporation of the solvent, the jet is solidified forming polymer fibers. Under the condition of invariable electrospinning parameters, when the THF content is less in the mixed solvent, the electrospinning solution has poorer volatility. This affects the phase separation of the solution in the process of jet stretching and, then, results in a decrease in the orientation and poorer mechanical properties of the polymer molecular chain. When the THF content is more in the mixed solvent, the electrospinning solution has better volatility. However, under the electric field force during electrospinning, it is unable to form a stable jet; the polymer molecular chains cannot entangle, resulting in a decrease in the mechanical properties of the polymer molecular chain.

\section{Conclusions}

PVDF is widely used because of its good properties, such as resistance to chemical corrosion and weatherability. PVDF/PEI composite fiber membranes were prepared using electrospinning and the properties of different solvents ratio
PVDF/PEI composite fiber membranes were tested. The conclusions are as follows:

(1) On the basis of the finite element calculation theory of electric field, the electric field distribution in a representative electrospinning device is computed. The electric field structure of a needle-plate type electrospinning device was simulated by means of ANSYS software. And the vector distribution of the nozzle on the spinneret pipe was got.

(2) SEM images show that when the THF ratio is too small or too large, the electrospinning solution is too slow, and the jet is still not solidified after deposition on the collection device, making it easy to form fiber bonding. When the electrospinning solution volatilized easily, it is difficult to form the Taylor cone at the nozzle, which is not conducive to form the fiber.

(3) X-ray diffraction analysis shows that under the influence of an electric field force the crystal form is transformed from $\alpha$ into $\beta$ and $\delta$ forms, and the PVDF with the $\beta$ crystal form has a strong ferroelectric effect during electrospinning. Therefore, it is expected that the PVDF/PEI nanometer composite material with ferroelectric function can be obtained by electrospinning. DSC curves show that under certain electrospinning parameters the volatility of the electrospinning solution has some influence on the orientation and alignment of the polymer molecular chains under the electric field force.

(4) Mechanical test results show that with the increase in the THF content the fracture elongation and the fracture stress of PVDF/PEI first increase and then decrease. When the solvent mass ratio was $\mathrm{m}(\mathrm{DMF}) / \mathrm{m}(\mathrm{THF})=7: 3$, the fracture stress and the fracture elongation of the composite electrospinning fiber films are maximum. This is because the increase in the THF content leads to an increase in the volatility of the spinning solution. Under the action of the electric field force, the molecular chain structure is improved, and the mechanical properties are improved. However, when the THF content is too large, the jet becomes unstable, which is not conducive to form fibers, and the mechanical properties decrease. These agree well with the DSC results.

\section{Competing Interests}

The authors declare that they have no competing interests.

\section{Acknowledgments}

This work is supported by Heilongjiang Postdoctoral Funds for Scientific Research Initiation (Grant no. LBH-Q13093), Science Funds for the Young Innovative Talents of HUST (Grant no. 201509), and Harbin Science and Technology Innovation Researchers Project (Grant no. 2012RFJGG013). 


\section{References}

[1] Y. Yang, Z. D. Jia, J. Liu et al., "Effect of electric field distribution uniformity on electrospinning," Journal of Applied Physics, vol. 103, no. 8, Article ID 104307, 2008.

[2] H. W. Duan, J. G. Jiang, B. Y. Li, B. Li, and T. H. He, "Electric field finite element analysis and experimentation of plate-plate type electrospinning machine," International Journal of Control and Automation, vol. 7, no. 7, pp. 179-190, 2014.

[3] H. Duan and J. Jiang, "Experimentation and finite element analysis of electric field structure of electrospinning machine," in Advances in Computer Science, Intelligent System and Environment, D. Jin and S. Lin, Eds., vol. 105 of Advances in Intelligent and Soft Computing, pp. 283-289, Springer, Berlin, Germany, 2011.

[4] A. Khoshnevis, S. S. H. Tsai, and E. Esmaeilzadeh, "Electric field induced sheeting and breakup of dielectric liquid jets," Physics of Fluids, vol. 26, no. 1, Article ID 012103, 2014.

[5] Y. Zheng, S. Xie, and Y. Zeng, "Electric field distribution and jet motion in electrospinning process: from needle to hole," Journal of Materials Science, vol. 48, no. 19, pp. 6647-6655, 2013.

[6] Q. L. Jiang, D. Z. Hu, M. Q. Jia, and R. S. Xue, "Effect of heat treatment temperature on the electrochemical properties of SnSb-based Cu electrodes for lithium batteries," Applied Surface Science, vol. 312, pp. 109-115, 2014.

[7] O. Karatay, M. Dogan, T. Uyar, D. Cokeliler, and I. C. Kocum, "An alternative electrospinning approach with varying electric field for 2-D-aligned nanofibers," IEEE Transactions on Nanotechnology, vol. 13, no. 1, pp. 101-108, 2014.

[8] E. Jentzsch, Ö. Gül, and E. Öznergiz, "A comprehensive electric field analysis of a multifunctional electrospinning platform," Journal of Electrostatics, vol. 71, no. 3, pp. 294-298, 2013.

[9] C. J. Angammana and S. H. Jayaram, "The effects of electric field on the multijet electrospinning process and fiber morphology," IEEE Transactions on Industry Applications, vol. 47, no. 2, pp. 1028-1035, 2011.

[10] X. Wang, X. Wang, and T. Lin, "3D electric field analysis of needleless electrospinning from a ring coil," Journal of Industrial Textiles, vol. 44, no. 3, pp. 463-476, 2014.

[11] Z.-X. Liu, H.-Y. Li, W.-F. Wu, H.-B. Chen, Y.-M. Ding, and W.M. Yang, "Effect of electric field on gas-assisted melt differential electrospinning with hollow disc electrode," Journal of Polymer Engineering, vol. 35, no. 1, pp. 61-70, 2015.

[12] R. S. Xue, H. Liu, P. P. Wang, and Z. M. Shen, "Formation of nanocarbons during activation of mesocarbon microbeads with potassium hydroxide," Carbon, vol. 47, no. 1, pp. 318-320, 2009.

[13] Y. S. Zheng and Y. C. Zeng, "Electric field analysis of spinneret design for multihole electrospinning system," Journal of Materials Science, vol. 49, no. 5, pp. 1964-1972, 2014.

[14] Y. S. Zheng, R. S. Xie, and Y. C. Zeng, "Electric field distribution and jet motion in electrospinning process: from needle to hole," Journal of Materials Science, vol. 48, no. 19, pp. 6647-6655, 2013.

[15] J.-G. Jiang, H.-W. Duan, T.-H. He, and B. Li, "Electric field simulation and experimentation of needle-plate type electrospinning machine," Journal of Computational and Theoretical Nanoscience, vol. 12, no. 9, pp. 2016-2022, 2015.

[16] I. M. Alarifi, A. Alharbi, W. S. Khan, A. Swindle, and R. Asmatulu, "Thermal, electrical and surface hydrophobic properties of electrospun polyacrylonitrile nanofibers for structural health monitoring," Materials, vol. 8, no. 10, pp. 7017-7031, 2015.

[17] S. A. Memon, W. Liao, S. Yang, H. Cui, and S. F. A. Shah, "Development of composite PCMs by incorporation of paraffin into various building materials," Materials, vol. 8, no. 2, pp. 499518,2015

[18] W. S. Khan, R. Asmatulu, and M. M. Eltabey, "Electrical and thermal characterization of electrospun PVP nanocomposite fibers," Journal of Nanomaterials, vol. 2013, Article ID 160931, 9 pages, 2013.

[19] S.-B. Yang, C. Han, X.-K. Wang, and M. Nagatsu, "Characteristics of cesium ion sorption from aqueous solution on bentoniteand carbon nanotube-based composites," Journal of Hazardous Materials, vol. 274, pp. 46-52, 2014.

[20] S. Ramakrishna, K. Fujihara, W. Teo, T. Lim, and Z. Ma, An Introduction to Electrospinning and Nanofibers, World Scientific, Danvers, Mass, USA, 2005.

[21] J. H. Wendorff, S. Agarwal, and A. Greiner, Electrospinning: Materials, Processing, and Applications, Wiley Online Library, Hoboken, NJ, USA, 2012.

[22] K. M. Yun, C. J. Hogan Jr., Y. Matsubayashi, M. Kawabe, F. Iskandar, and K. Okuyama, "Nanoparticle filtration by electrospun polymer fibers," Chemical Engineering Science, vol. 62, no. 17, pp. 4751-4759, 2007. 

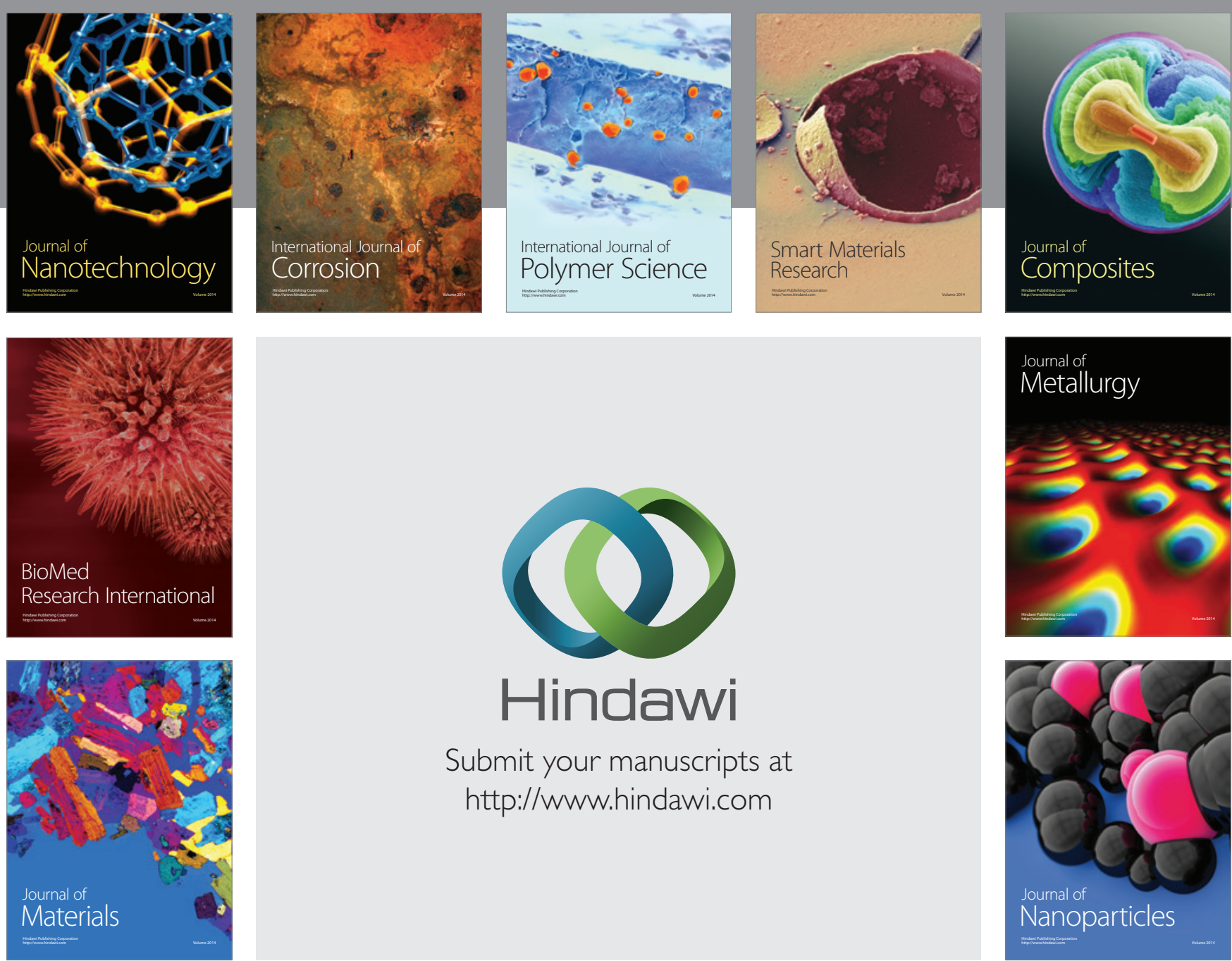

\section{Hindawi}

Submit your manuscripts at

http://www.hindawi.com

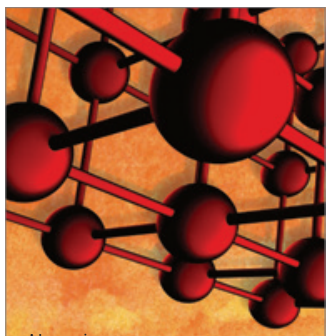

Materials Science and Engineering
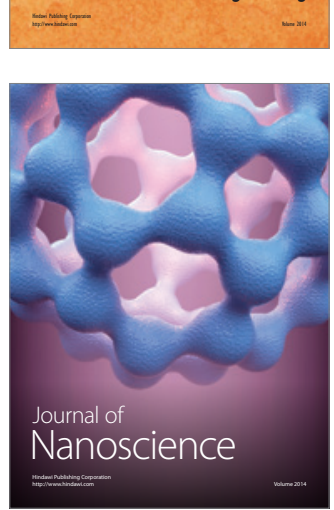
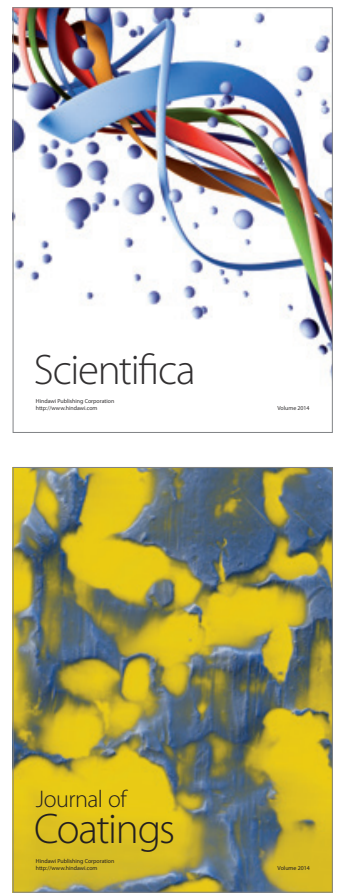
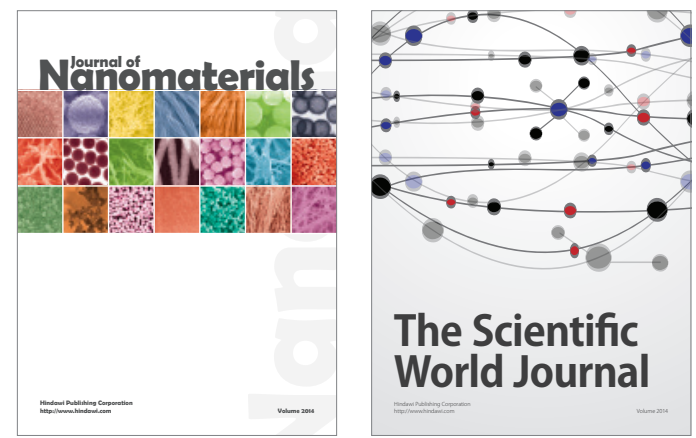

The Scientific World Journal
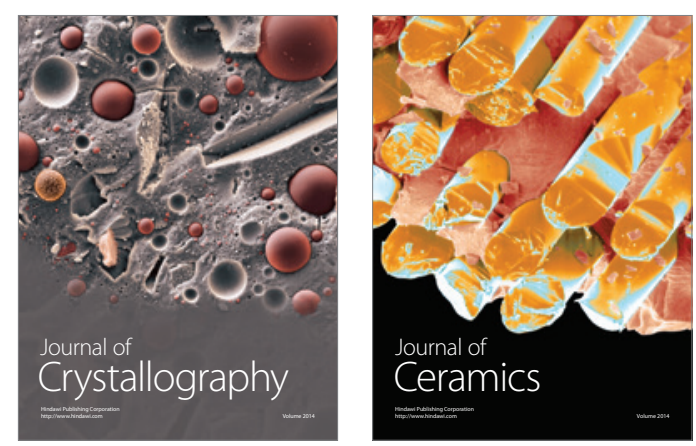
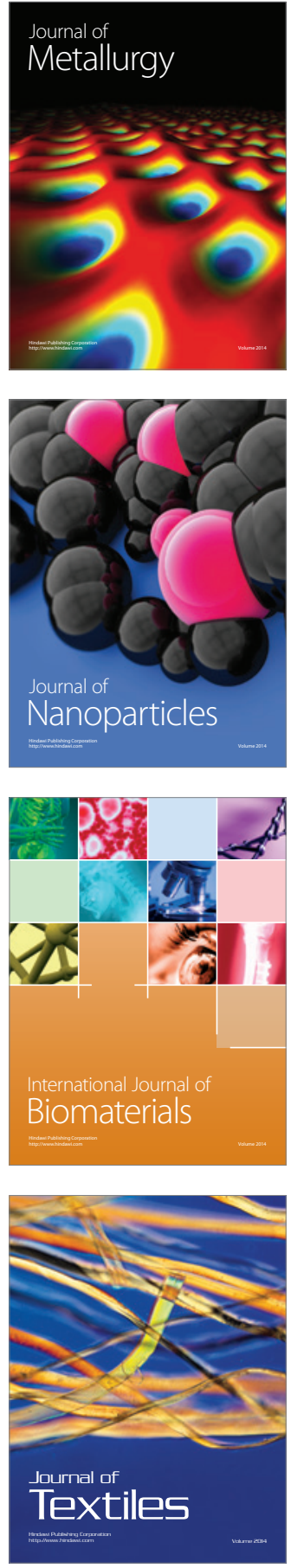